\title{
Watershed of a continuous function
}

\author{
Laurent Najman ${ }^{a, b}$ and Michel Schmitt ${ }^{a}$ \\ ${ }^{a}$ L.C.R, Thomson-CSF, Domaine de Corbeville, 91404 Orsay, France. \\ Tel: (33-1) 60197782 / Fax : (33-1) 60197120 \\ E-mail : najman@thomson-lcr.fr \\ ${ }^{b}$ CEREMADE, Université Paris Dauphine, 75775 Paris, France.
}

\begin{abstract}
The notion of watershed, used in morphological segmentation, has only a digital definition. In this paper, we propose to extend this definition to the continuous plane. Using this continuous definition, we present the watershed differences with classical edge detectors. We then present a metric in the plane for which the watershed is a skeleton by influence zones and show the lower semicontinuous behaviour of the associated skeleton. This theoretical approach suggests an algorithm for solving the eikonal equation: $\|\nabla f\|=g$. Finally, we end with some new watershed algorithms, which present the advantage of allowing the use of markers and/or anchor points, thus opening the way towards grey-tone skeletons.
\end{abstract}

\section{Résumé}

La notion de ligne de partage des eaux, utilisée en segmentation morphologique dispose uniquement d'une définition digitale. Dans cet article, nous proposons d'étendre la définition de la ligne de partage des eaux au plan continu. En utilisant cette définition continue, nous comparons la ligne de partage des eaux avec les extracteurs de contours classiques, et montrons leurs différences. Nous introduisons ensuite une métrique pour laquelle la ligne de partage des eaux est un squelette par zones d'influence, ce qui nous permet de montrer son comportement semi-continu. Cette approche théorique nous suggère un nouvel algorithme pour résoudre l'équation d'eikonal : trouver $f$ telle que $\|\nabla f\|=g$. Nous terminons enfin sur de nouveaux algorithmes de ligne de partage des eaux, présentant l'avantage de pouvoir inclure des 
marqueurs et des points d'ancrages, ouvrant ainsi la voie aux squelettes à teintes de gris. 


\section{Introduction}

One of the most intuitive notions of morphological segmentation is the notion of watershed $[3,13,22,2,28]$. The algorithms proposed in the literature have only a formal link with the various definitions of the watershed. Intuitively, the idea is that the watershed is a skeleton by influence zones with respect to a special distance, but all the previous theoretical definitions are in the general case a kind of skeleton, i.e. the previous watersheds do have barbs.

In this paper, we propose a proof of the convergence of the algorithm of Beucher and Lantuéjoul [3]. This proof allows us to give a meaning to a continuous definition of the watershed, and to show the very link between the watershed and the skeleton. Using this continuous definition, we are able to compare the watershed with the classical second order differential operators used to detect edges. This theoretical work leads us to new algorithms, one to solve the eikonal equation, and the other one to compute a watershed, with the advantage of allowing the use of markers and the use of anchor points.

\section{The watershed: from discrete to contin- uum}

\subsection{Classical digital algorithm}

We follow here the presentation of L. Vincent [28].

In mathematical morphology, it is usual to consider that an image is a topographical surface. It is done by considering the grey level (the image intensity) as an altitude. Places of high variation in the intensity are then a good set in which one can search for contour lines. It is then rather straightforward to estimate the variation from the gradient of the image. For the purpose of segmentation, we are then looking for the crest lines of the gradient image. A way of doing this operation is to apply the watershed algorithm to the gradient image.

The idea of the watershed is to attribute an influence zone to each of the regional minima of an image (connected plateau from which it is impossible to reach a point of lower grey level by an always descending path). We then define the watershed as the boundaries of those influence zones.

Numerous techniques have been proposed to compute the watershed. The majors ones are rewied in $[28,30]$. The classical idea for building the watershed is simple to describe in one dimension (fig. 1). We begin by piercing the 


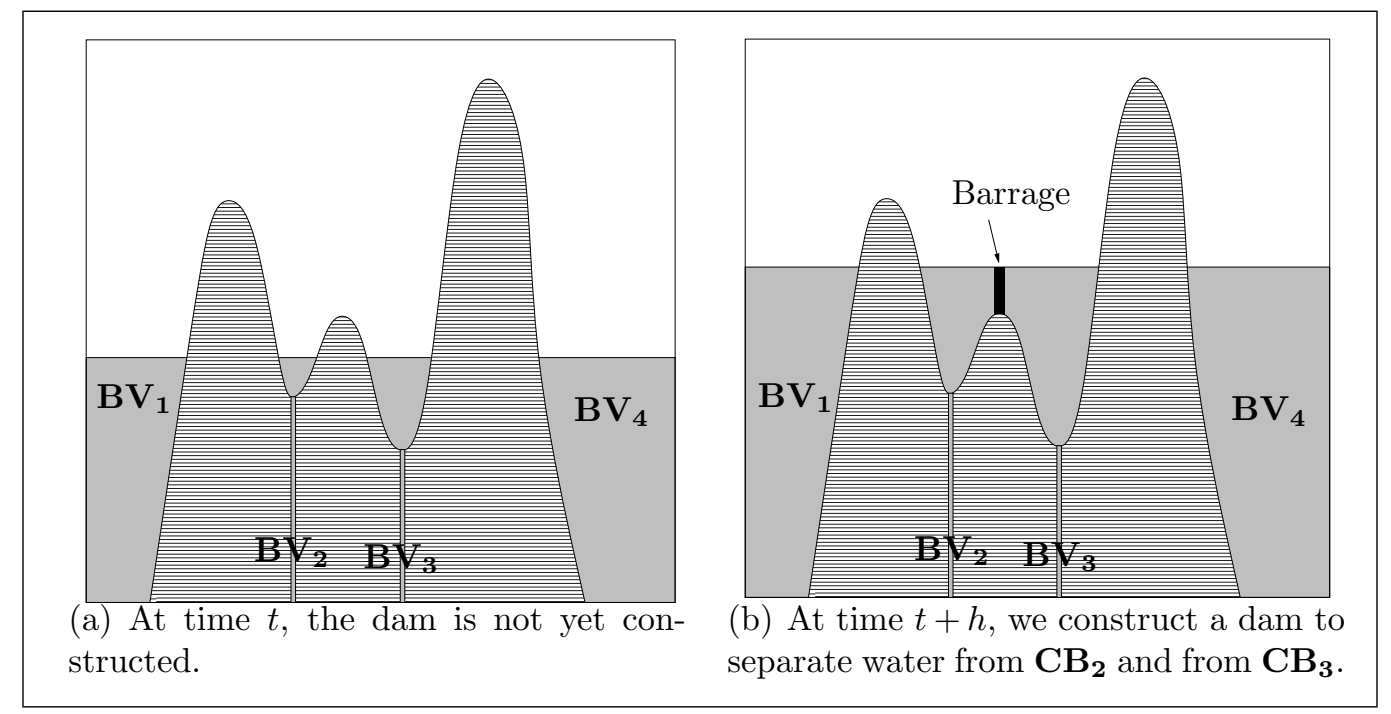

Figure 1: Building of the watershed: one-dimensional approach

regional minima of the surface. Then, we slowly immerse the image into a lake. The water progressively floods the basins corresponding to the various minima (fig. 1.a). To prevent the merging of two different waters originating from two different minima, we erect a dam (fig. 1.b). Once the surface is totally immersed, the set of the dams thus built is the watershed of the image. In one dimension, the location of the watershed is straightforward. In two dimension (which is the case of the classical images) this characterization is not so easy. One can say in an informal way that the watershed is the crest lines of the image.

We give here the classical algorithm allowing the computation of the watershed. The most powerful implantation described in the literature [30, 29, 4, 21] uses FIFO breadth-first scanning techniques for the actual flooding.

Following the ideas mentioned above, the algorithm consists in flooding the water in the various basins, and in keeping as the watershed the set of contact points between two different basins. In the case where this contact is on a plateau, we keep the (geodesic) middle line of this plateau. The watershed thus defined is of thickness one on the grid.

To compute the geodesic middle on the contact plateaus, we use the geodesic distance.

Definition 2.1: Let $A$ be a set, $a$ and $b$ two points of $A$. We call geodesic distance in $A d_{A}(a, b)$ the lower bound of the lengths of the paths $\gamma$ in $A$ linking $a$ and $b$. 


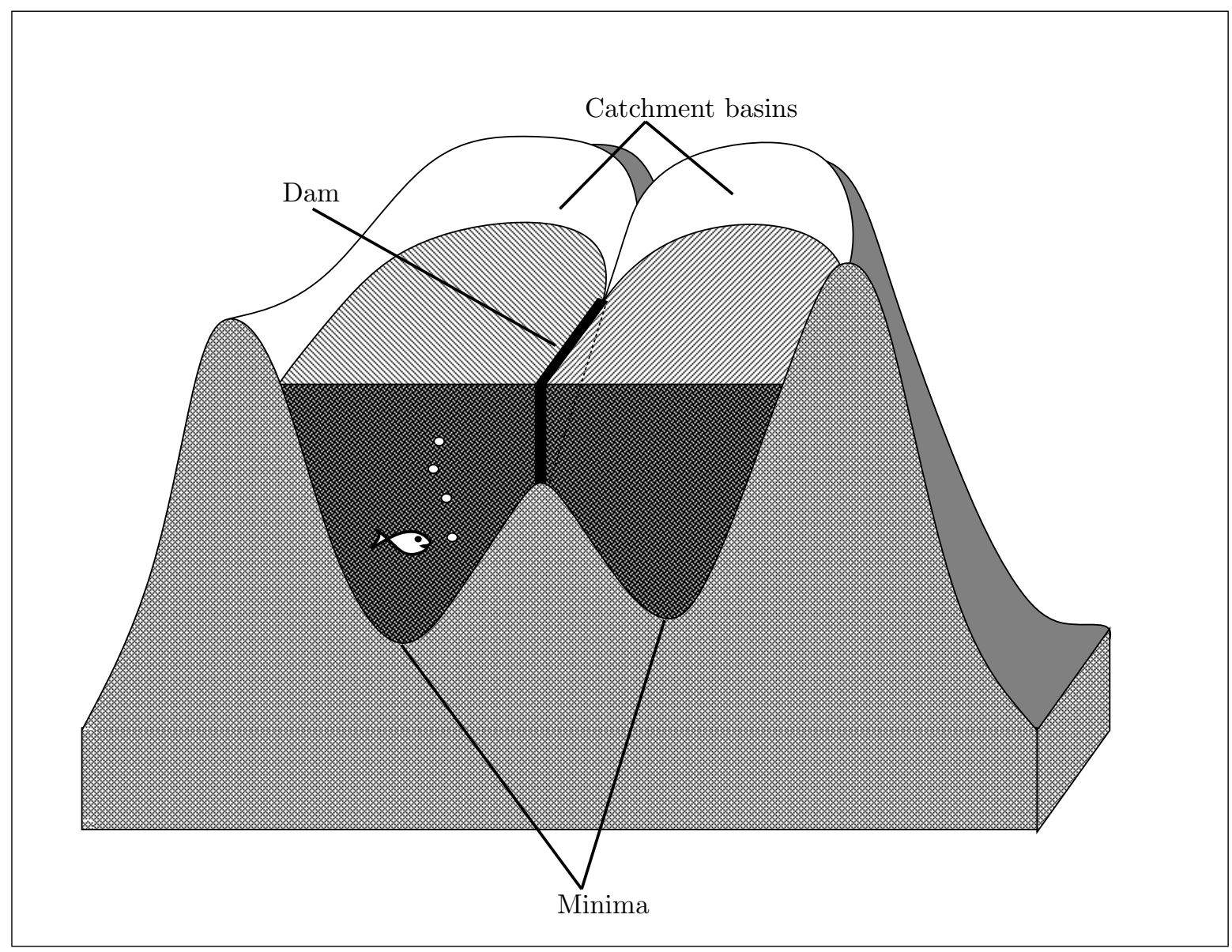

Figure 2: Building of the watershed in 2D

In the digital case, the distance $d_{A}$ is deduced from the one on the grid [20].

Let $B=\cup B_{i} \subset A$, where $B_{i}$ are the connected components of $B$.

Definition 2.2: The geodesic influence zone $i z_{A}\left(B_{i}\right)$ of a connected component $B_{i}$ of $B$ in $A$ is the set of the points of $A$ for which the geodesic distance to $B_{i}$ is smaller than the geodesic distance to other connected components of $B$.

$$
i z_{A}\left(B_{i}\right)=\left\{p \in A, \forall j \in[1, k] \backslash\{i\}, \quad d_{A}\left(p, B_{i}\right)<d_{A}\left(p, B_{j}\right)\right\} .
$$

The points of $A$ which do not belong to any influence zone make up the skeleton by influence zones of $B$ in $A$, noted $S K I Z_{A}(B)$ :

$$
S K I Z_{A}(B)=A \backslash I Z_{A}(B)
$$

where $I Z_{A}(B)=\bigcup_{i \in[1, k]} i z_{A}\left(B_{i}\right)$. 
The watershed algorithm on digital images by recurrence on grey level is [3]:

Definition 2.3: The set of the catchment basins of the numerical image I is the set $X_{h_{\max }}$ obtained after the following recurrence:

(i) $\quad X_{h_{\min }}=T_{h_{\min }}(I)$

(ii) $\forall h \in\left[h_{\min }, h_{\max }-1\right], X_{h+1}=\operatorname{Min}_{h+1} \cup I Z_{T_{h+1}(I)}\left(X_{h}\right)$.

where:

- $h_{\min } \in \mathbb{Z}$ (resp. $h_{\max }$ ) is the lowest (resp. the greatest) grey level of image $I$.

- $T_{h}(I)$ is the threshold of the image $I$ at height $h: T_{h}(I)=\{p \mid I(p) \leq h\}$

- Min ${ }_{h}$ is the set of the regional minima of $I$ at the height $h$.

The watershed of the image I is the complement of this set.

Note that this algorithm works only for step functions.

\subsection{Continuous generalization}

From now on, the image $f$ is supposed to be regular enough $\left(\mathcal{C}^{2}\right)$ to allow the use of differential operators. We use classical tools of differential geometry [24]. The gradient $\nabla f$ is the plane vector of the first order derivatives of $f$, and the Hessian $H_{f}$ is the real symmetric matrix of the second order derivatives.

The definitions of watershed are based on the notion of path of greatest slope. Intuitively, a path of greatest slope is a path parallel to the gradient of $f$. If we begin on a point $a$ where $\nabla f(a) \neq 0$, we can easily follow the gradient line backward (i.e. the grey-levels are decreasing along the line) until we reach a point $b$ where $\nabla f(b)=0$. But $b$ is not necessarily a regional minima of $f$ and thus there is an ambiguity to continue the path after $b$. We propose to formalize this notion by using the notion of maximal line of the gradient. A path of greatest slope will then be an union of maximal lines of the gradient.

Definition 2.4: A path $\gamma:]-\infty,+\infty\left[\rightarrow \mathbb{R}^{2}\right.$ is called a maximal line of the gradient if

$$
\begin{gathered}
\forall s \in]-\infty,+\infty[, \dot{\gamma}(s)= \pm \nabla f(\gamma(s)) \neq 0 \\
\text { and } \lim _{s \rightarrow-\infty} \dot{\gamma}(s)=\lim _{s \rightarrow+\infty} \dot{\gamma}(s)=0
\end{gathered}
$$

We shall say that a maximal line of the gradient is descending if

$$
\forall s \in]-\infty,+\infty[, \dot{\gamma}(s)=-\nabla f(\gamma(s))
$$


The maximal lines of the gradient are defined on ] $-\infty,+\infty$ [ for one cannot reach a point with a zero speed. This is due to the parametrisation (the speed at which we run on the path) we have chosen. The magnitude of this speed is equal to the gradient modulus. We could have chosen another parametrisation of the path, but we use it as it expresses the fact that we cannot clearly extend a maximal line of the gradient. Note that the union of all maximal lines of the gradient of a continuous function covers the whole domain of the function (as an example, see fig.4).

We recall that $a$ is a critical point if $\nabla f(a)=0$. We need to link the maximal lines of the gradient if we want them to end in a regional minima. We are going to define a partial ordering relation which will allow us to do so.

Definition 2.5: Let $a$ and $b$ be two critical points of $f$. We shall say that $b$ $i s$ above $a$ if there exists a maximal descending line of the gradient linking $b$ to $a$. We can extend this notion by saying that $b$ is above $a$ if there exists a set $\left(a_{i}\right)$ of critical points such that $a_{0}=b$ and $a_{n}=a$, satisfying $a_{i}$ is above $a_{i+1}$. We then obtain the partial ordering relation "above".

This ordering relation allows to distinguish three kinds of critical points:

- regional minima,

- points above an unique minima

- points above several minima

The last ones should clearly belong to the definition of the watershed of a continuous function.

Definition 2.6: We denote by $\mathcal{P}(f)$ the subset of the critical points a of $f$ which are above several regional minima of $f$.

Fig. 3 shows some examples of points of $\mathcal{P}(f)$.

We have the following convergence theorem which will be used in the following as our definition of the continuous watershed:

Theorem 2.7: [17] Let $f$ be a $\mathcal{C}^{2}$ function, with a compact connected domain. Suppose that $f$ has only isolated critical points, and that, on the critical points, the Hessian has two non zero eigenvalues. We construct a sequence $f_{n}$ of step functions which converges pointwise towards $f$. More precisely, we put $f_{n}(a)=\frac{E\left(2^{n} f(a)\right)}{2^{n}}$, where $E(x)$ is the integer part of $x$. Then, the watershed of $f$, seen as the limit of the watershed of $f_{n}$, is the set of the maximal lines of the gradient linking two points of $\mathcal{P}(f)$. 


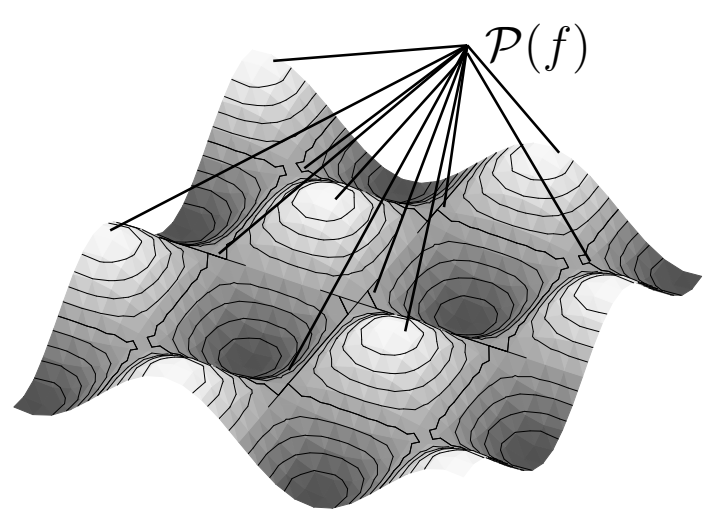

Figure 3: Some examples of points of $\mathcal{P}(f)$

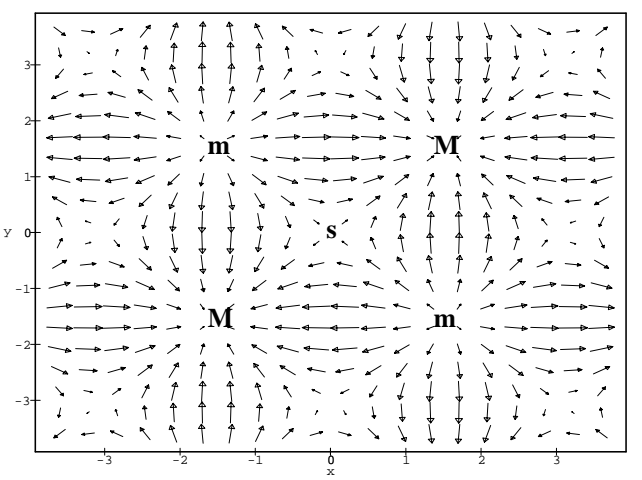

Figure 4: Gradient field of $\sin (x) \sin (y)(\mathrm{M}$ : maxima - m: minima - s: saddle point)

Note that the limit function $f$ does not have plateaus. In fact, we will show in the next section that the choice of a line of watershed on a plateau is arbitrary.

So the watershed lines are parallel to the gradient, which, as far as we know, has not been pointed out by other authors. Note that we can use theorem 2.7 as a definition of the watershed of a continuous function. One should be very careful with the hypothesis we put on $f$. If Hessian $H_{f}$ has one zero eigenvalue, the watershed can have a barb (a branch with an endpoint), which is never the case with the algorithm. As an example, one can look at a monkey saddle (fig. 5). Two branches of the monkey saddle belong to the contour of a basin. The other one direct itself towards a local maximum of the image which belongs to the interior of a basin. Moreover, the watershed of a continuous function can be thick. As an example, we 
can look for what Beucher [2] calls a buttonhole (see fig. 6). Choosing a particular line in the buttonhole is, anyway, fully arbitrary. Note that, on the buttonhole, $\nabla f(a)=0$ yields $H_{f}(a)=0$. Nevertheless, we can always assume that the images satisfy the hypothesis of the theorem 2.7: if $f$ does not satisfy it, then an arbitrary small perturbation of $f$ makes $f$ an adequate function (obviously the perturbation must not vanish on the critical points). Such functions are called Morse functions [16], and are (uniformly) dense in the set of smooth functions.

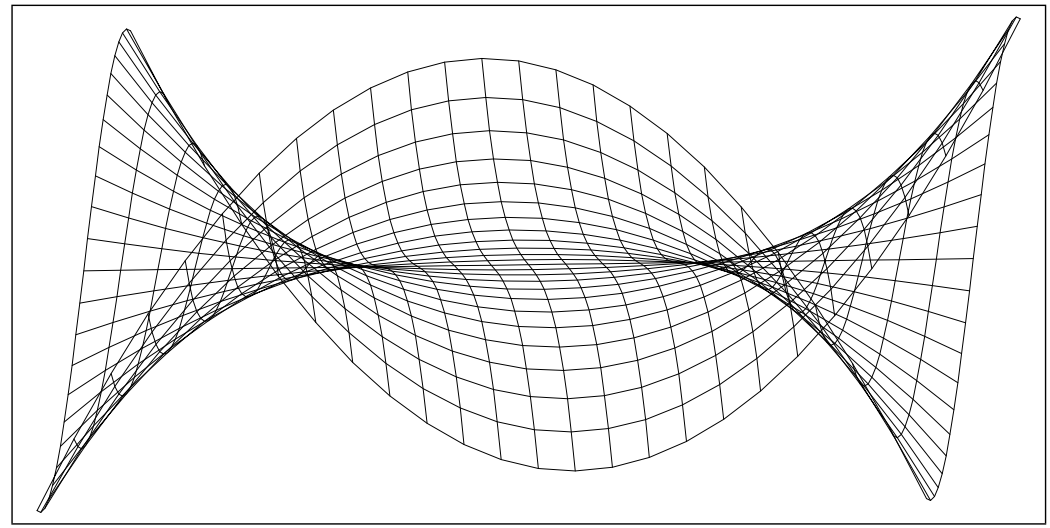

Figure 5: Monkey saddle

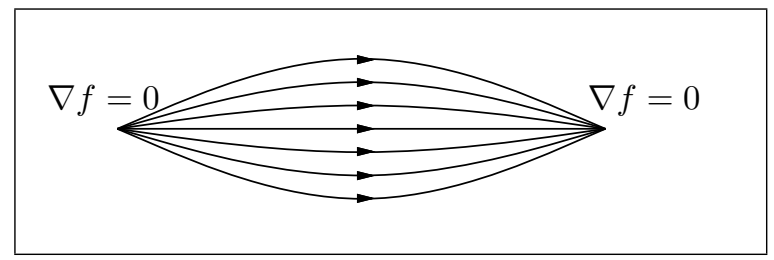

Figure 6: Gradient field of a buttonhole

The fact that at each point of a $\mathcal{C}^{2}$ function there exist at least 2 lines of greatest slope descending towards two minima is true only on points of $\mathcal{P}(f)$ (see fig. 4).

The theorem 2.7 suggests that we can add some lines to the watershed by adding points to $\mathcal{P}(f)$. If we carefully choose these new points, the result exhibits end points and is a way to introduce the notion of grey-tone skeleton. In the last part of this paper we give an algorithm which allows to do such an operation.

To define the watershed as a subset of the maximal lines of the gradient is a local notion, to which boundary conditions add a global aspect. More precisely, we have the following proposition: 
Proposition 2.8: [17] Let a be a point of the domain of $f$ such that $\nabla f(a) \neq$ 0 . Let $\mathcal{V}_{a}$ be a neighbourhood of a which does not contain any critical point. Let $\gamma$ be a path containing $a$ and parallel to the gradient of $f$ on $\mathcal{V}_{a}$. Then there exists a function $f_{0}$, equal to $f$ on $\mathcal{V}_{a}$, such as $\gamma$ is in the watershed of $f_{0}$.

In other words, there is no local characterization of the watershed. This is due to the $\mathcal{C}^{2}$ regularity of $f$. If $f$ is less regular, there exists in some cases a local characterization. The best example is the watershed of $f(a)=d(a, X)$ where $X$ is a binary image. In this case the watershed of $f$ is equal to the skeleton by influence zones of $X$. As shown by Matheron [23], it is locally characterized by the set of the points of non differentiability of $f$ : the watershed is included in this set of points but not always equal to it.

\subsection{The problem of the plateaus}

Real images often possess plateaus. When they belong to the interior of a catchment basin, there is no problem. From a theoretical point of view, it is easy to slope the plateaus towards crest lines without modifying the watershed.

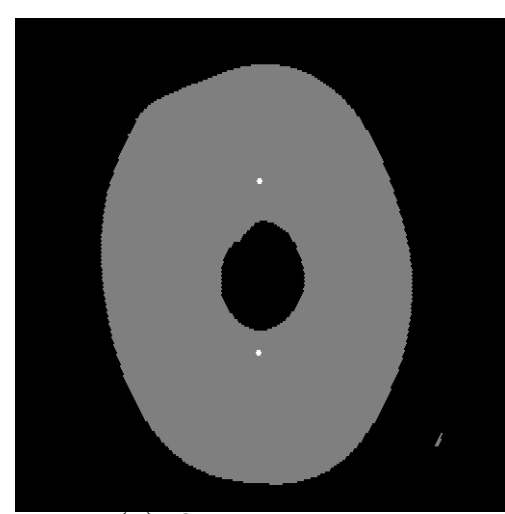

(a) Original image

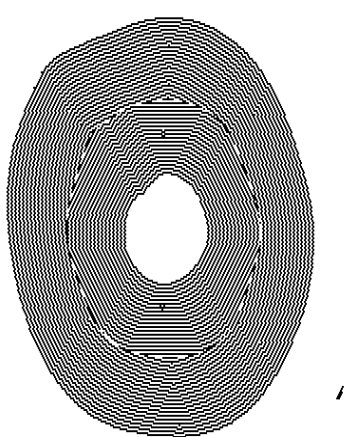

(b) Distance to the descending side

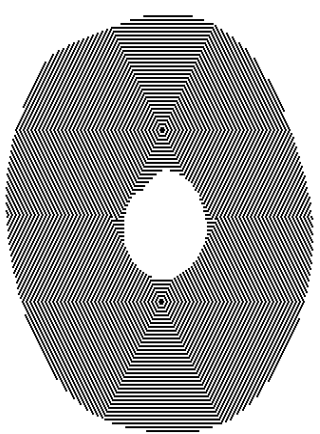

(c) Distance to the ascending side

Figure 7: Distances to the sides of a ring with two elevated points near the interior side

On the contrary, if we want a thin watershed, we have to make a choice on the plateaus. The immersion algorithm 2.3 chooses a line by computing the "geodesic middle" of the plateaus. The principle is to compute the geodesic distance to the descending side of each plateau (fig. 7.b). There exists another 
possibility: it is possible to compute the geodesic distance to the ascending side of each plateau (fig. 7.c). These two possibilities give different results, and the choice of one rather than the other is arbitrary and depends on the application. We give here some examples illustrating the choice of the distance.

Let us take the image of a ring, corresponding to the contours of an object. The watershed of this image reduces the ring to the middle line, thus creating two equal parts, which seems reasonable. Nevertheless, there are some situations where we want the segmentation to pass through another place.

Let us consider the figure 8.a and the figure 8.d. They are the ring image on which we have added some new contour points through which we want the watershed to pass. In the first case (fig. 8.a), these points are near the exterior side, and on the second case (fig. 8.d), these points are near the interior side. The watershed immersion algorithm floods the image of the distance to the descending side (fig. 8.c and fig. 8.f), which do not seem a judicious choice.

If we transform the image by computing the distance to the ascending side (the distance to the added points), the watershed result then conforms to the intuition on the figure 8.b. There exists no reasonable criterion which allows a choice between the figures 8.e and 8.f: there are not enough points to correctly place the contour.

These examples are a good illustration that the implicit choice of the distance to the descending side is fully arbitrary, for it can be judicious to choose the distance to the ascending side. The distance to the descending side is nevertheless the right choice in the case where we want the watershed to pass exactly at the middle of the plateaus.

Let us notice that the use of the distance to the descending side is unstable: it can add some new local minima, thus modifying the topology of the watershed. As an example, there exists a tendency to close arcs of circle (fig. 9). On the other side, the distance to the descending side creates new local maxima, but this does not disturb the watershed.

\section{Comparison with the edge detectors}

Let $f$ be a smooth function. Two second order differential operators are commonly used to detect edges. The first one is the Laplacian [14] $\Delta f=$

$\frac{\partial^{2} f}{\partial x^{2}}+\frac{\partial^{2} f}{\partial y^{2}}$ and the second one is the non-linear Canny's detector [5] which looks for the maximum of the gradient in the direction of the gradient. The Canny's 


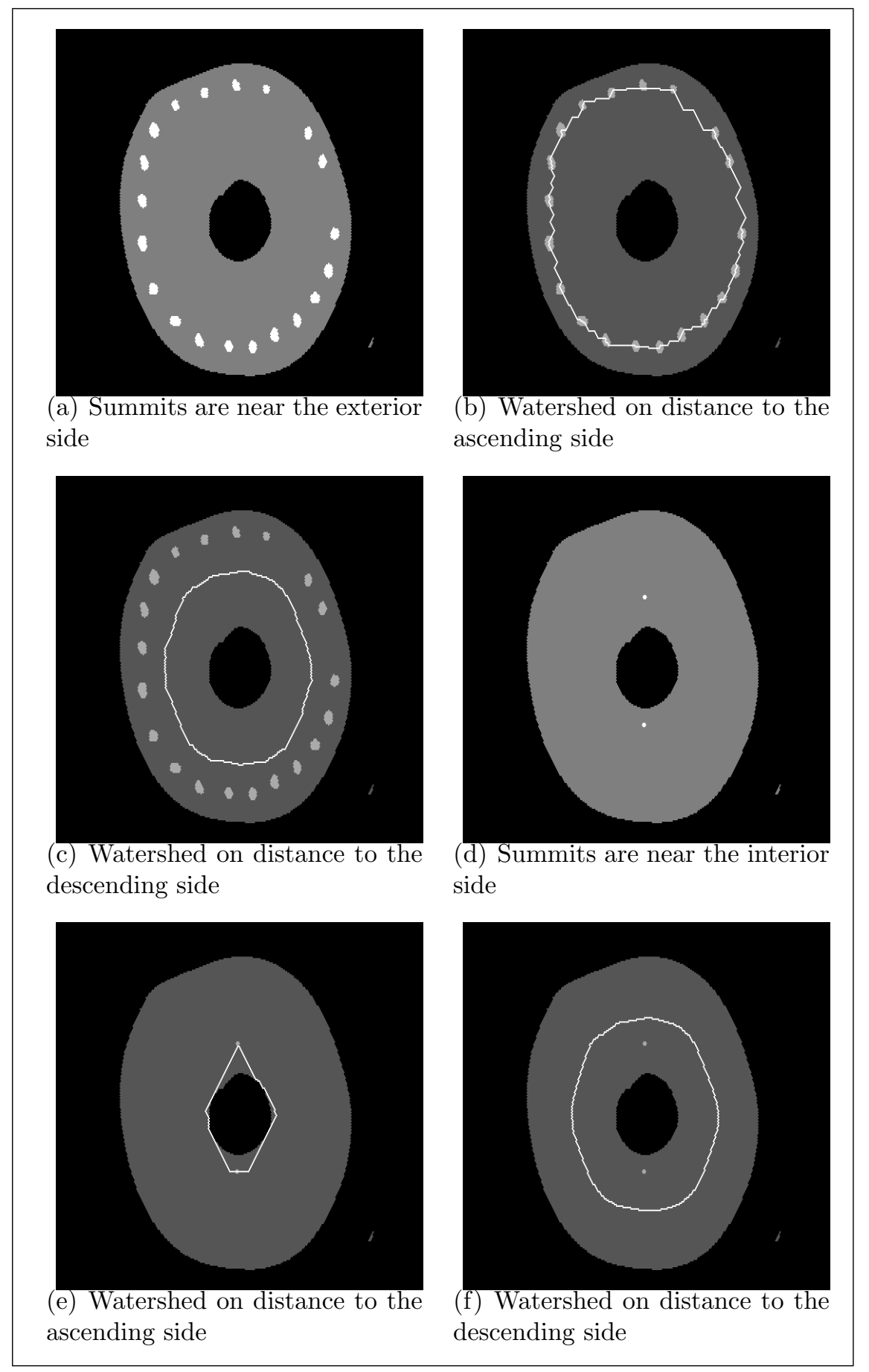

Figure 8: plateaus' problem: the choice of a well-placed contour 


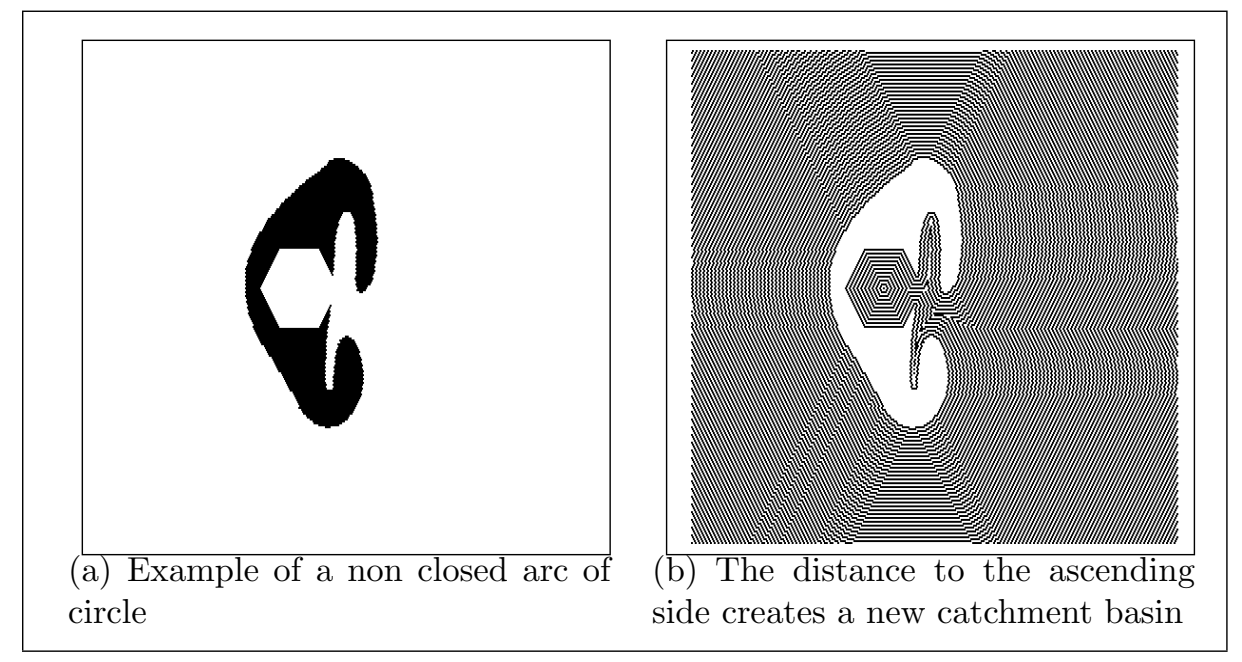

Figure 9: Example of creation of a new catchment basin through the distance to the ascending side

detector, or more exactly the extrema of the gradient in the direction of the gradient, finds the zero crossings of $Q(f)=\left\langle H_{f} \nabla f, \nabla f\right\rangle$. On the other hand, mathematical morphology uses the watershed of the norm of the gradient of $f$ in order to extract edges. The links between the two differential operators are well known [25] and we focus our attention on the link between Canny's detector and the watershed of the gradient.

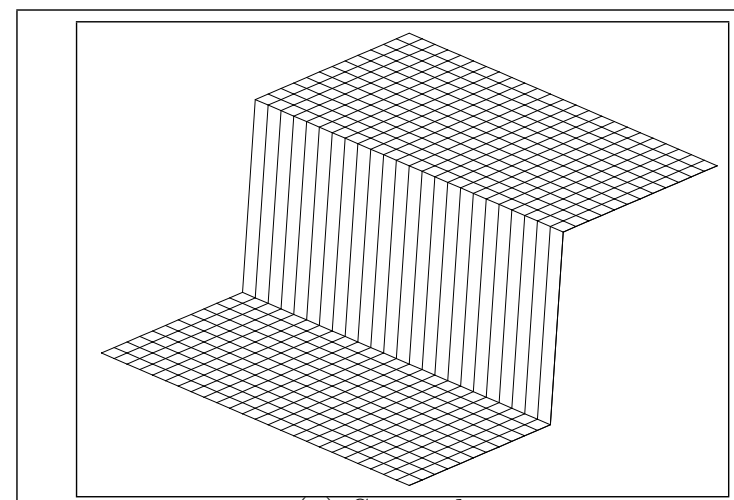

(a) Step edge

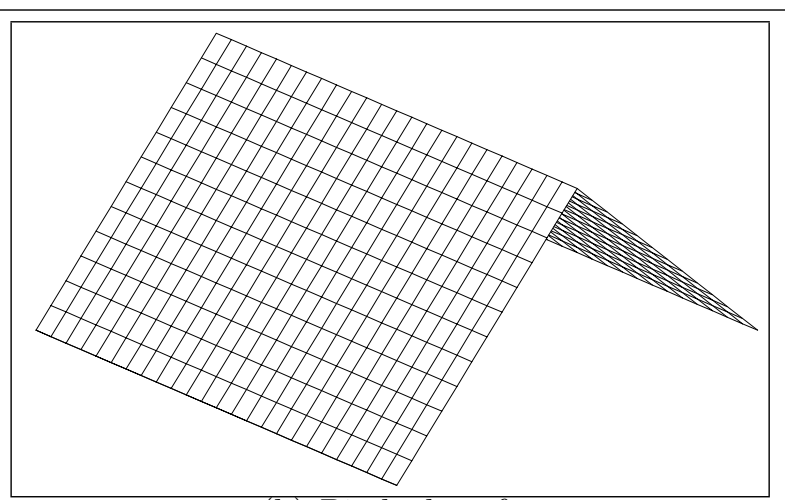

(b) Pitched roof

Figure 10: Structural archetype detected by Canny's operator

In [11] the authors exhibit a characterization of lines extracted by Canny's detector. Their results are useful to point out the differences between the watershed of the gradient and the second order differential operators. There are two archetypes of structure on which $Q$ vanishes: the step edge and the 
pitched roof. These two objects are represented on figure 10, before the gaussian convolution which makes them smooth.

The idea behind edge detection is that an edge is a path where the change in the intensity $f$ is maximum in the direction normal to this path. As the intensity is computed by the modulus of the gradient, we can write

$$
\frac{d}{d t}\|\nabla f(a+t n)\|=\left\langle H_{f} \nabla f, n\right\rangle=0
$$

where $n$ is the normal to the path at point $a$. This equation gives an implicit differential equation for the edge path: $\dot{\gamma}=H_{f} \nabla f$. As we have seen before, the union of all the paths which are solutions to this differential equation covers the whole domain of $f$, and we have to make a choice to find the edges. The watershed chooses the paths by imposing boundary conditions. On the other hand, Canny solves the problem by estimating the normal $n$ from the gradient direction, i.e. by setting $n=\nabla f$ (which is true on a step edge).

We made a comparison of the action of these operators on the image

$$
I(x, y)=\delta_{1} \chi_{\{x>0\}}(x, y)+\delta_{2} \chi_{\{y>0\}}(x, y)
$$

where $\chi_{A}$ is the characteristical function of the set $A$ : $\chi_{A}(a)=1$ if $a \in A$ and $\chi_{A}(a)=0$ if not. $I$ is regularised by a gaussian kernel $\mathrm{G}$ and we obtain

$$
f=G * I=\delta_{1} \Psi(x)+\delta_{2} \Psi(y)
$$

where $\Psi(x)=\frac{1}{\sqrt{\pi}} \int_{-\infty}^{x} e^{-s^{2}} d s$.

Fig. 11 shows the comparison of the segmentation algorithms. We see that Canny's detector finds the multiple point only if $\delta_{1}=\delta_{2}$ (fig. 11.f).

Fig. 12 shows the results: the second order operators cannot find the multiple point, while the watershed of the gradient modulus can. This is due to the geometric behavior of Canny's detector and zeros of the Laplacian. Both are the intersection of a function $z=g(x, y)$ with $\{z=0\}$. So, they have very few multiple points. On the other hand, the watershed has multiple points which are necessarily in $\mathcal{P}(\|\nabla f\|)$.

Several technics [7, 8, Giraudon \& Deriche] have been recently developed to detect the multiple points. They are based on second-order differential measures and scale-space approach. In fact, we propose a simpler method: if we are interested in finding multiple points, the classical differential crest extractor (local maxima of the modulus of the gradient in the direction of the gradient) has to be replaced by a watershed procedure on the image of gradient modulus. 


\section{Metrical approach of the watershed}

The aim of this section is to exhibit the strong link between the skeleton, one of the notions of the binary mathematical morphology, and the watershed, notion of the grey-level mathematical morphology.

Definition 4.1: The image distance on a $\mathcal{C}^{1}$ function $f$ with a connected domain Dom $(f)$, is defined by: $\forall(a, b) \in \operatorname{Dom}(f)^{2}$,

$$
d_{f}(a, b)=\inf _{\gamma_{a b}}\left|\int_{\gamma_{a b}}\left\|\nabla f\left(\gamma_{a b}(s)\right)\right\| d s\right|
$$

Note that the shortest $d_{f}$-path between $a$ and $b$ is a path of greatest slope if it exists.

We restrain the choice of $f$ to the $\mathcal{C}^{2}$ functions which have only isolated critical points. $d_{f}$ is then a distance. For technical reasons, but without loss of generality, we suppose that the minima of $f$ are on the same level. Moreover, we suppose that on the critical points, the Hessian has two non zero eigenvalues. We then have the following result:

Theorem 4.2: [17] The set of points which are at equal $d_{f}$-distance of two distinct minima of $f$ is the set of the maximal lines of the gradient linking two points of $\mathcal{P}(f)$, and thus coincides with the watershed of $f$.

A similar result has been stated in [19, 18, Preteux\&Merlet], but with a much more complex metric used as a definition for the continuous watershed, and this complex metric is very far from the usual euclidean distance. Note that the watershed defined by Lantuéjoul and Beucher [3], the one defined by Maisonneuve [13], the one defined by Preteux and Merlet, and the $d_{f^{-}}$ skeleton by influence zones do have barbs if $f$ does not verify the hypothesis we put on it.

The advantage of our metric is to allow the statement of new results we present hereafter. Moreover, note that if we put $\|\nabla f\|=1$ a.e., $d_{f}$ (or $f$ ) is the usual euclidean distance function to a set, and the $d_{f}$-skeleton by influence zones is the usual one.

With the results of the previous theorem, we can expect that the watershed has properties similar to those of the skeleton. We state one of these properties.

We denote by $\mathcal{M}$ the set of minima of $f$ and by $\operatorname{Dom}(f)$ the domain of $f$.

Definition 4.3: We define the skeletal structure of $f$ as the set of centers of the maximal open $d_{f}$-balls contained in $\operatorname{Dom}(f) \backslash \mathcal{M}$. 
Obviously, the watershed is contained in the skeletal structure.

Theorem 4.4: [17] The mapping $f \rightarrow \mathcal{S}(f)$, where $\mathcal{S}(f)$ is the skeletal structure of $f$, is lower semicontinuous, if we use the $\mathcal{C}^{2}$ convergence on the set of functions and the induced hit or miss topology [15] on the set of watersheds.

This result shows why the watershed is very sensitive to noise, and justifies in a way the various smoothing [9] and marking [28] techniques.

\section{The eikonal equation}

As a side effect, the algorithm of the watershed can be adapted to solve an equation widely used in Shape from Shading, the eikonal equation [10]:

$$
\text { finding } f \text { such as }\|\nabla f\|=g
$$

The idea is that, on each catchment basin of $f$, we have $f(a)=d_{f}(a, b)+f(b)$, where $b$ is the minima of the catchment basin. As determining $d_{f}(a, b)$ only depends on $g$ (see formula 9 ), we can generalize this result: let $\left\{b_{i}\right\}$ be a set of points with their associated values $f\left(b_{i}\right)$. A continuous solution to the eikonal equation is given by

$$
f(a)=\inf _{i}\left\{\inf _{\gamma_{a b_{i}}} \int_{\gamma_{a b_{i}}} g\left(\gamma_{a b_{i}}(s)\right) d s+f\left(b_{i}\right)\right\}
$$

One can show [12] that the function $f$ given by equation 11 is the unique viscosity solution satisfying $\|\nabla f\|=g$ on an open domain $\Omega$, which is a viscosity supersolution on $\partial \Omega$.

The algorithm proposed by Vincent [28] can be adapted to compute this solution. In fact, Vincent's algorithm splits up in a first stage of sorting the pixels by increasing grey level, and a second stage of flooding propagation threshold by threshold. In our case, we can't make the first stage, but we can do the sorting during the flooding. This is easy to do if we use an heapsort algorithm [1]. We then obtain a kind of algorithm similar to the one developed by Verwer and Verbeek [27, 26].

Note that this technique can also be used to compute the watershed by flooding from selected sources, an efficient tool to prevent the oversegmentation problem. 


\section{Towards grey-tone skeleton}

The theorem 2.7 suggests the possibility of adding some points to $\mathcal{P}(f)$, and thus some lines to the watershed. This lines will then figure the contours inside the basins. We give a new algorithm to compute the watershed which allows to do this.

This algorithm is based on Vincent's one for building skeleton with anchor points [28]. The idea is to do an homotopic thinning of each grey level of the image. We then compute the skeleton by influence zones of the level $h-1$ in the level $h$ by homotopic thinning. This algorithm gives us a watershed.

This implementation has two advantages. The first one is that it is easy to add anchor points : it is enough to prevent the suppression of some points. The second one is that instead of flooding from the minima of the image, we can flood from any marker we want.

Figure 13 shows an example of application of this new algorithm.

The main problem is the choice of the anchor points. A first idea is to use the segmentation provided by a classical edge detector, as Canny's one. We then have the multiple points which cannot be found by those algorithms, and we keep the edge lines interior to the basins.

Another idea of anchor points is to generalize the notion of binary skeleton. An interesting class of anchor points is the set of the centers of osculating circles to the descending sides of plateaus. On a binary image, these points are in the skeleton of the form. If we transform our image by computing the distance to the descending side of the plateaus, the set of the points of the following (hexagonal) configuration:

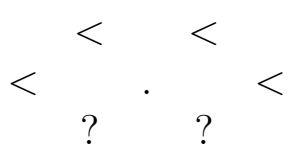

(where a $<$ marks a pixel the value of which is lower than the value of the central pixel, and where? means that this value is unimportant) contains these centers, and is thus a good choice for the anchor points. In particular, if we use this procedure on the distance function of a binary image, we obtain the usual skeleton of thickness one.

Figure 14 shows various possibility of segmentation on the image of a face sideview (fig. 14.b). The first one (fig. 14.a) is the classical Canny-Deriche edge detector [6]. The second one (fig. 14.b) is obtained by the classical watershed algorithm applied to the gradient image. The result is very noisy, but contains all the useful information. The third one (fig. 14.d) is obtained by the classical watershed algorithm applied to a geodesical reconstruction 
of gradient image of size 10, which is a useful technique to suppress a lot of unimportant local minima [9]. The fourth and last one (fig. 14.e) is obtained by the new watershed algorithm applied to a geodesical reconstruction of gradient image of size 10, using the Canny-Deriche edges as anchor points.

\section{Conclusion}

This paper is mainly devoted to the convergence and adaptation to the continus case of an algorithm defined for step functions. This gives us a mathematical tool which links the watershed to the notion of line of greatest slope (maximal line of the gradient), and to the notion of skeleton by influence zones. The associated skeleton is then lower semicontinuous.

The watershed is compared with the classical edge detectors, and we showed that the watershed can extract multiple points, an operation that second order differential operators are unable to perform.

On the algorithmic side, the watershed characterization gives an original interpretation of the eikonal equation, and opens the path towards greytone skeletons. The powerful watershed-by-flooding algorithm offers efficient adaptations to these various notions.

\section{References}

[1] A. Aho, J. Hopcroft, and J. Ullmann. Data Structures and Algorithms. Addison-Wesley Publishing Company, Inc., Reading, Mass., 1983.

[2] S. Beucher. Segmentation d'images et morphologie mathématique. Thèse École Nationale Supérieure des Mines de Paris, June 1990.

[3] S. Beucher and Ch. Lantuéjoul. Use of watersheds in contour detection. In Proc. International Workshop on Image Processing, Real-Time Edge and Motion Detection/Estimation, Rennes, september 1979.

[4] S. Beucher and F. Meyer. The Morphological Approach to Segmentation: The Watershed Transformation. In E.R. Dougherty, editor, Mathematical Morphology in Image Processing, Optical engineering, pages 433-482. Marcel Dekker, inc., New York - Basel - Hong Kong, 1993.

[5] J.F. Canny. A computational approach to edge detection. In M.A. Fischler and O. Firschein, editors, Readings in Computer Vision: Issues, Problems, Principles and Paradigms, pages 184-203. Morgan Kaufmann Publishers, Inc., 1986. 
[6] R. Deriche. Using Canny's Criteria to Derive a Recursive Implemented Optimal Edge Detector. International Journal of Computer Vision, pages 167-187, 1987.

[7] Giraudon, G. and Deriche, R. Accurate corner detection: an analytical study. Technical Report 1420, INRIA, April 1991.

[8] Giraudon, G. and Deriche, R. On corner and vertex detection. Technical Report 1439, INRIA, June 1991.

[9] M. Grimaud. La Géodésie Numérique en Morphologie Mathématique : Application à la Détection Automatique de Microcalcifications en Mammographie Numérique. Thesis, École des Mines de Paris, December 1991.

[10] B.K.P. Horn. Robot Vision. The MIT Engineering and Computer Science Series. The MIT Press, Mac Graw-Hill Book Company, 1986.

[11] W.M. Krueger and K. Phillips. The Geometry of Differential Operators with Application to Image Processing. IEEE PAMI, 11:1252-1264, December 1989.

[12] P.L. Lions, E. Rouy, and A. Tourin. Shape-from-shading, viscosity solutions and edges. Numerische Mathematik, 64:323-353, 1993.

[13] F. Maisonneuve. Sur le partage des eaux. Technical report, CGMM, École des Mines de Paris, 1982.

[14] D. Marr and E.C. Hildreth. Theory of edge detection. In Proc. Roy. Soc. Lond. B, pages 187-217, 1980. Vol. 207.

[15] G. Matheron. Random Sets and Integral Geometry. John Wiley and Sons, New York, 1975.

[16] J. Milnor. Morse Theory. Princeton University Press, 1963.

[17] L. Najman and M. Schmitt. Quelques caractérisations de la ligne de partage des eaux d'une fonction continue. Technical Report ASRF-924, L.C.R., Août 1992.

[18] F. Prêteux. On a distance Function Approach for Gray-level Mathematical Morphology. In E.R. Dougherty, editor, Mathematical Morphology in Image Processing, Optical engineering, pages 323-350. Marcel Dekker, inc., New York - Basel - Hong Kong, 1993.

[19] F. Prêteux and N. Merlet. New concept in Mathematical Morphology: the Topographical and Differential Distance functions. In Image Algebra and Morphological Image Processing II, volume 1568, pages 66-77, San Diego, California, July 1991. 
[20] M. Schmitt. Some examples of algorithms analysis in computational geometry by means of mathematical morphological techniques. In J.D. Boissonnat and J.P. Laumond, editors, Geometry and Robotics, pages 225-246. Lecture Notes in Computer Science, Vol. 391, Springer Verlag, 1989 .

[21] M. Schmitt and L. Vincent. Morphological image analysis: a practical and algorithmic handbook. Cambridge University Press, To appear in 1993.

[22] J. Serra. Image Analysis and Mathematical Morphology. Academic Press, London, 1982.

[23] J. Serra, editor. Image Analysis and Mathematical Morphology, Volume 2: Theoretical Advances. Academic Press, London, 1988.

[24] M. Spivak. A Comprehensive Introduction to Differential Geometry, volume 2. Publish Or Perish, Inc., Houston, Texas, second edition, 1979.

[25] V. Torre and T. Poggio. On edge detection. IEEE Transactions on Pattern Analysis and Machine Intelligence, PAMI-8:147-163, March 1986.

[26] B.J.H. Verwer. Distance Transforms: metrics, algorithms and applications. PhD thesis, Technical University Of Delft, The Netherlands, 1991.

[27] B.J.H. Verwer and P.W. Verbeek. Shading from shape, the eikonal equation solved by grey-weighted distance transform. Pattern Recognition Letters, 11:681-690, October 1990.

[28] L. Vincent. Algorithmes morphologiques à base de files d'attente et de lacets : Extension aux graphes. Thèse École des Mines de Paris, May 1990.

[29] L. Vincent. Morphological Algorithms. In E.R. Dougherty, editor, Mathematical Morphology in Image Processing, Optical engineering, pages 255-288. Marcel Dekker, inc., New York - Basel - Hong Kong, 1993.

[30] L. Vincent and P. Soille. Watersheds in digital spaces: an efficient algorithm based on immersion simulations. IEEE Transactions on Pattern Analysis and Machine Intelligence, Vol. 13(No. 6):583-598, 1991. 


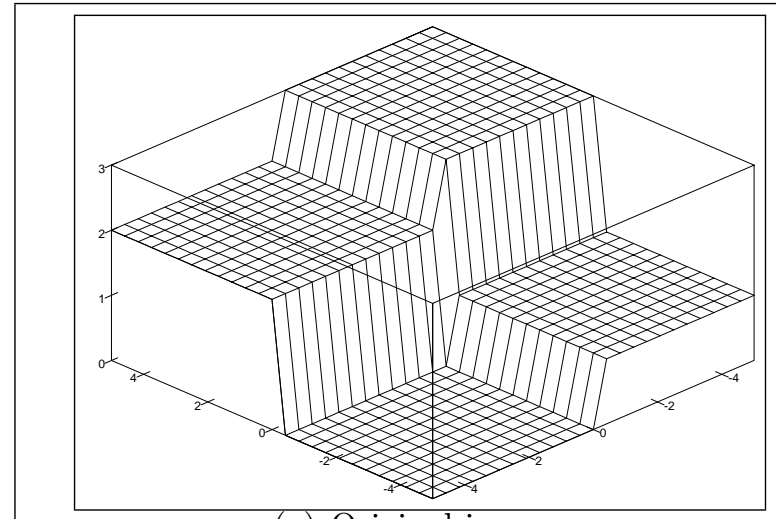

(a) Original image

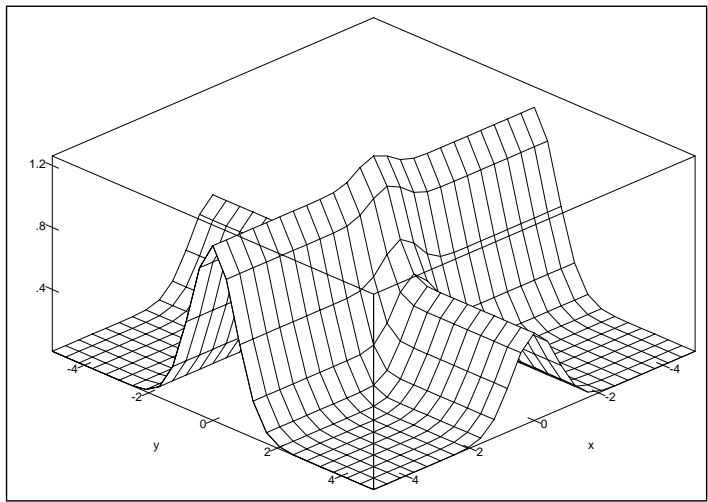

(c) Gradient norm

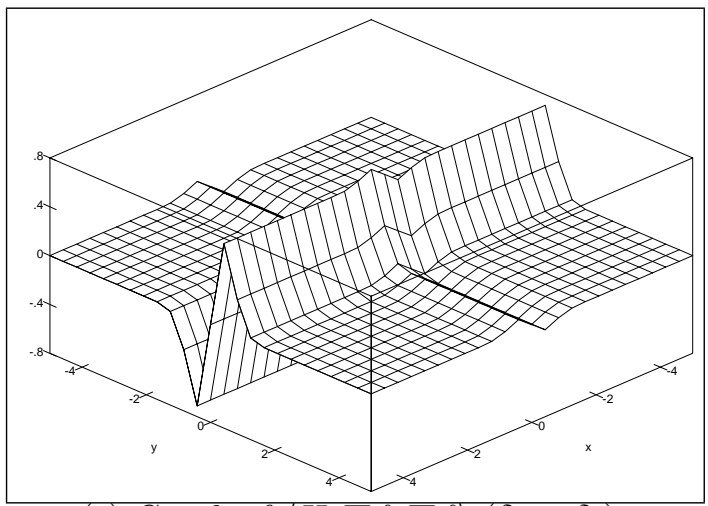

(e) Graph of $\left\langle H_{f} \nabla f, \nabla f\right\rangle\left(\delta_{1}<\delta_{2}\right)$

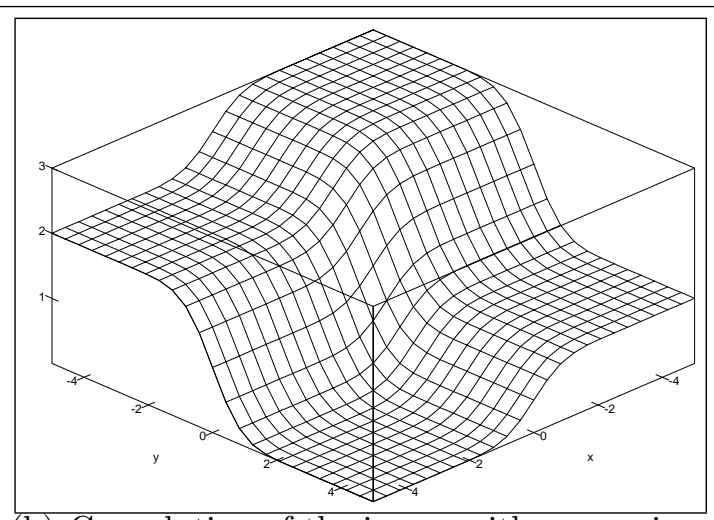

(b) Convolution of the image with a gaussian

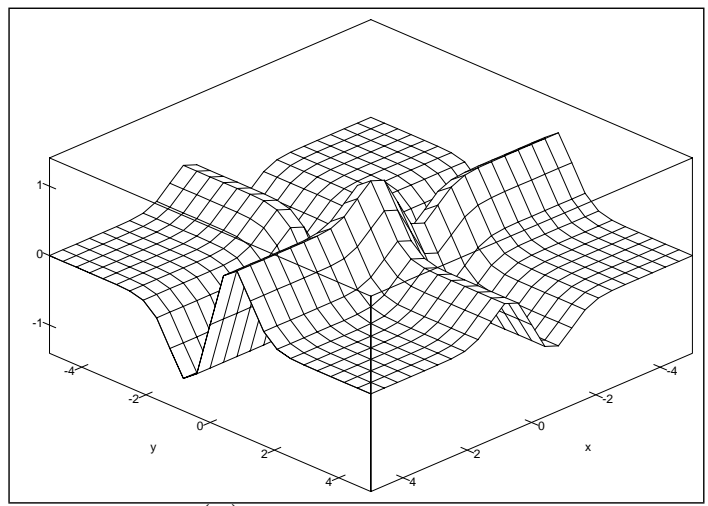

(d) Laplacian graph

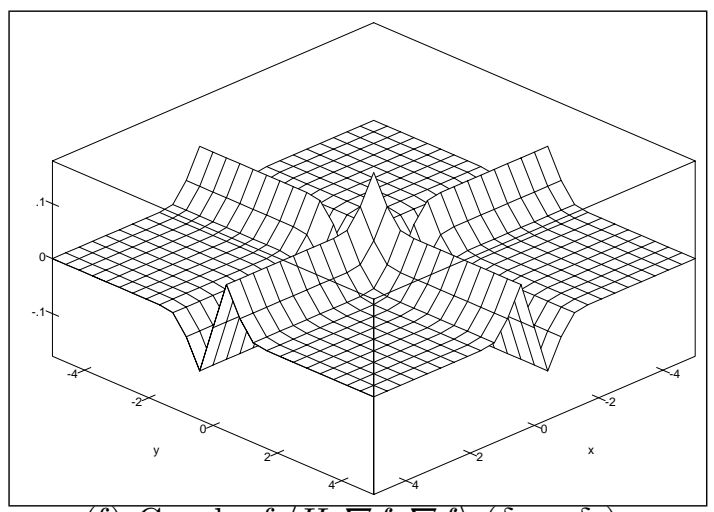

(f) Graph of $\left\langle H_{f} \nabla f, \nabla f\right\rangle\left(\delta_{1}=\delta_{2}\right)$

Figure 11: Comparison of the segmentation algorithms 


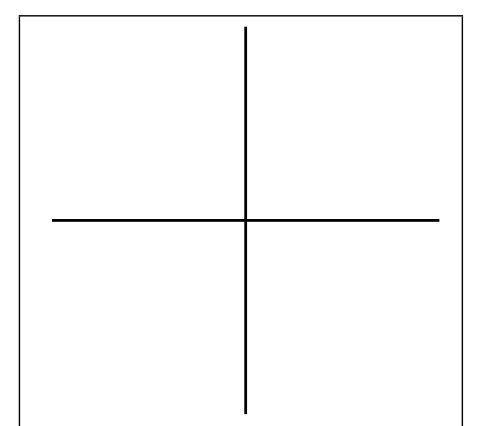

(a) Outlines found by watershed

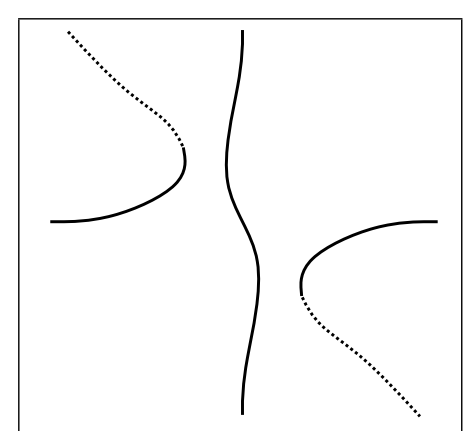

(b) Solution of $Q(f)=0$

Figure 12: Outlines found by the algorithms

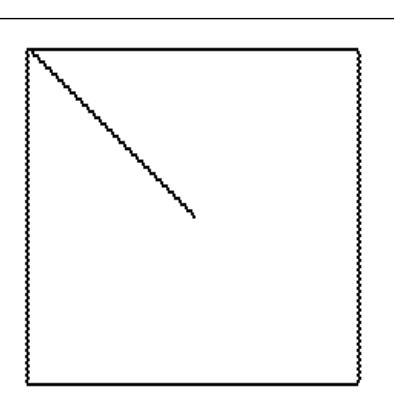

(a) Original image and result of the new watershed algorithm with the central points as anchor point

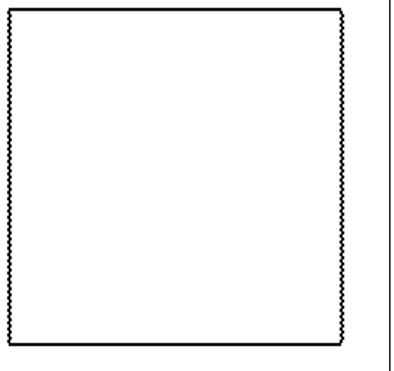

(b) Watershed on the image

Figure 13: An example of application of the new watershed algorithm 


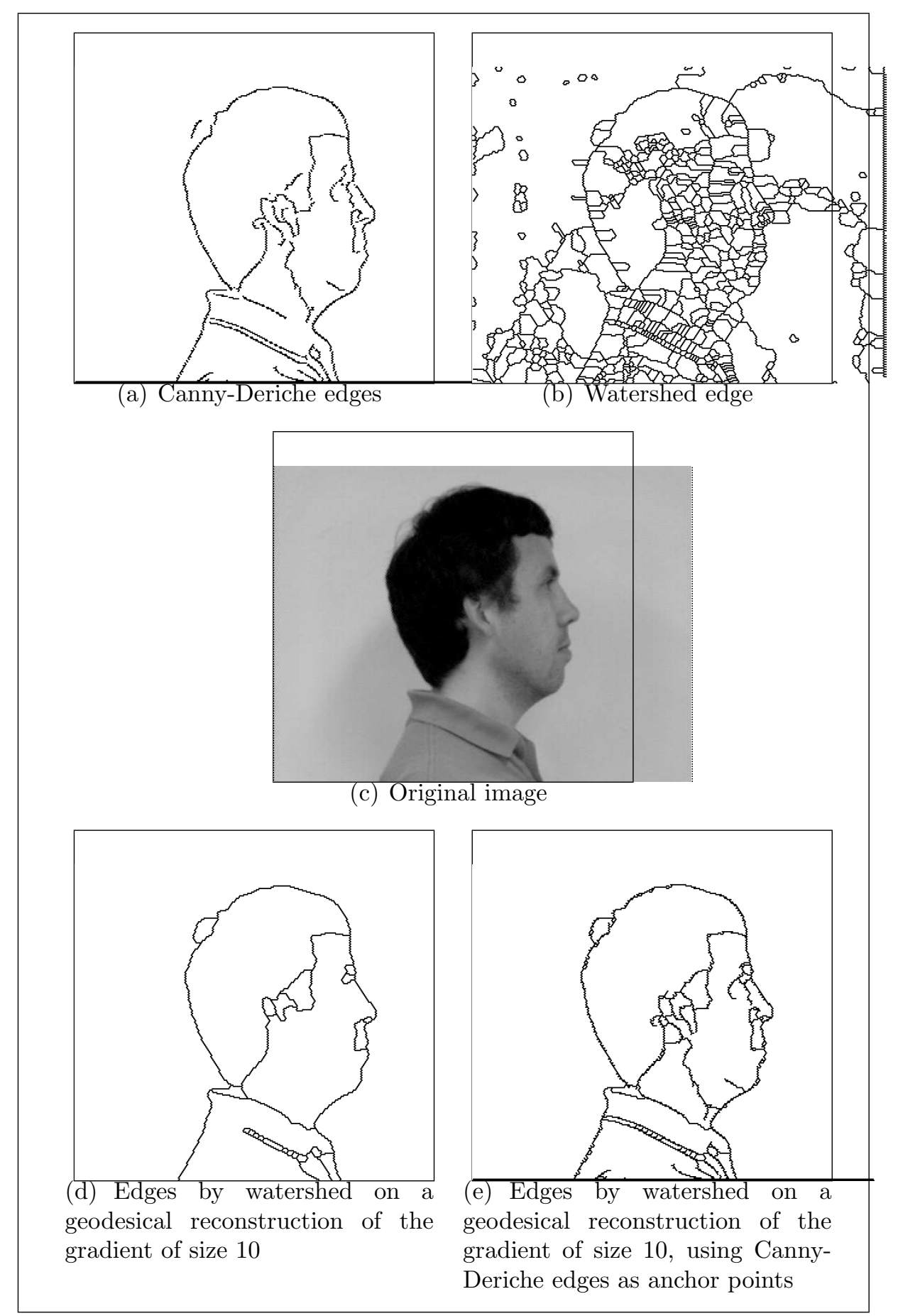

Figure 14: Comparison of various possibilities of segmentation 